\section{Axial Rotation of Globular Star Clusters}

THE axial rotation of globular star clusters has not yet been measured, so far as we know, from the differences of radial velocities at their opposite edges, but indirect evidence of their rotation is the observed ellipticity. The oblateness $e$ of a rotating body depends on the ratio $\varphi$ of the centrifugal force to the gravity at the star's surface; that is :

$$
e=C . \varphi, \text { where } \varphi=\omega^{2} r / g,
$$

and as has been proved by Clairaut ${ }^{1}$,

$$
\frac{1}{2} \varphi<e<\frac{5}{4} \varphi
$$

$\frac{1}{2}$ and $\frac{5}{4}$ being the limiting cases for the homogeneous and the centrally condensed stars. The constant $C$ as a function of the density distribution, that is, as a function of the polytrope class, was studied by H. N. Russell ${ }^{2}$ and recently very exhaustively by S. Chandrasekhar ${ }^{3}$.

The general outcome of theoretical investigations is in favour of the conclusion that the globular clusters are built on the model of a polytrope 4 or 5 . The constant $C$ changes only slightly within this interval, being 0.501 for $n=4$ and 0.500 for $n=5$. In consequence, the value $\frac{1}{2}$ can be accepted for the model of a globular cluster.

In this connexion, the observed ellipticities of the globular clusters are of interest. Generally, the observed value of the ellipticity is not the true one but depends on the orientation of the axis of rotation in space. For the sake of simplicity, we assume that the observed ellipticities are the true ellipticities, and as a mean value was adopted from Shapley's Catalogue of Globular Clusters, the error is insignificant. Ellipticities have been observed in many clusters ${ }^{4}$, and in some of them ellipticity shows throughout the cluster from centre to edge. The absolute dimensions of the clusters are approximately known but only scant information on their masses is available. We have adopted for the masses of the clusters $10^{5}-10^{7} \odot$, and the angular velocities were computed for the different values. Referring to our first equations, it may be noted that

$$
\omega^{2}=2 \gamma \frac{M e}{r^{3}},
$$

where $\gamma$ is the gravitational constant, $M$ the mass and $r$ the radius of the cluster. According to Shapley's determinations, the mean value of the oblateness was assumed to be $0 \cdot 123$, and for the mean diameter of a globular cluster $r=1.078 \times 10^{20} \mathrm{~cm}$. was used. The rotational velocity was calculated as a function of the mass, and the results are as follows :

$\begin{array}{lc}\begin{array}{c}\text { Mass } \\ =1\end{array} & \text { Period in years } \\ 1 \times 10^{8} \odot & 3 \cdot 877 \times 10^{8} \\ 2 \times 10^{5} & 2 \cdot 743 \times 10^{8} \\ 5 \times 10^{8} & 1 \cdot 735 \times 10^{8} \\ 1 \times 10^{8} & 1 \cdot 227 \times 10^{8} \\ 5 \times 10^{8} & 5 \cdot 487 \times 10^{7} \\ 1 \times 10^{7} & 3.877 \times 10^{7}\end{array}$

It follows that the mean period of rotation of a typical globular star cluster is approximately $10^{8}$ years. This value was deduced on the assumption that the globular clusters are rotating as rigid bodies, which cannot be said to be exactly true, because stars at different distances from the centre of the cluster evidently will have different rotational velocities. The problem is somewhat complicated by the presence of stars in different stages of evolution, but there is a possibility of determining (by star counts) the oblateness and therefore the different rotational velocities for different spectral classes. Such counts have been made by E. Freundlich and V. Heiskanen ${ }^{5}$ for $M$ 13. They find that the oblateness increases along the $B-M$ sequence. This interesting result interpreted from the present point of view means that the ratios of angular velocities for cluster stars of different spectral classes are:

$$
B: A: F:(G-K-M)=1.00: 1.40: 1.48: 1.70 ;
$$

that is, the stars of the $G-K-M$ types (giants) rotate twice as rapidly as stars of the $B$ type. It would be of great interest to know how far the above results are in agreement with the conditions in our local star cluster.

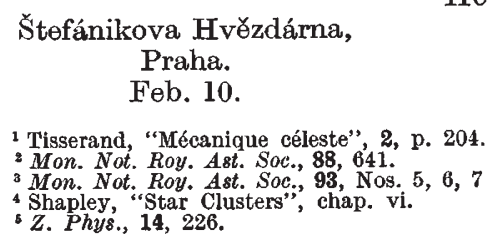

\section{Fire-Walking}

MANY of those taking part in the discussion on recent fire-walking performances appear to assume that a normal person would be burnt if his passage over the hot surface were identical with that of the fire-walker, and that a beginner can walk on charcoal or boulders with the same evenness and speed as a trained man. Having thus begged the question, they proceed to offer a variety of explanations, discordant in themselves, and ranging from thickened skin to an extrusion of ectoplasm ; thus illustrating once more the futility of propounding theories in the absence of vital experimental data.

As some observers may have a future opportunity of investigating a fire-walk, I would suggest the following tests, based on my experience at the recent performance of Kuda Bux :

(1) Fasten a small thermocouple to any part of the performer's foot which comes into contact with the hot charcoal or stone and connect it to a suitable indicator. The temperature attained by his skin may then be read at the moment he leaves the fire, and if the indicator were watched during the transit it could be noticed whether the same part of the foot was used at each contact. The duration of the contacts and the interval between each should be measured by a stopwatch.

(2) Measure the temperature of the hot surface by pressing on it a thermocouple constructed for this purpose, and connected to a second coil on the indicator which will enable this temperature to be read. Laboratory tests could then be made by raising a surface to this temperature and noting the effect on the skin of a normal subject when applied to the surface for the same periods, and with the same intervals, as those observed in the case of the firewalker.

(3) Strike the surface of the fire with the palm of the hand, making the same number of contacts as either of the performer's feet and with the same intervals between. If the observer feels that he is 